\title{
A relação entre a adesão e a evasão de idosos em projetos e atividades físicas ${ }^{1}$
}

\author{
The relationship between the membership and the \\ elderly evasion with projects and physical activities
}

João Coutinho Barroso Júnior²

Alfredo Gomes de Faria Junior ${ }^{2}$

\section{RESUMO}

O estudo observou a evasão de idosos nos projetos de atividade física da Secretaria de Esporte e Lazer, Cidade de Rio das Ostras. A motivação é o que leva o idoso a se matricular em programas de atividades físicas. Após a adesão, o peso da motivação vai fazer com que ele permaneça ou não na atividade. A evasão de idosos em atividades físicas já foi alvo de poucos estudos, o foco das pesquisas está mais direcionado à motivação para a adesão. $\mathrm{O}$ campo de pesquisa foram os projetos 'Bom Dia' e 'Hidroginástica'. Pesquisa quanti-qualitativa indicou uma relação entre a motivação e a evasão, a principal causa da evasão é a mesma que motiva a matrícula, a saúde ou a falta dela.

\section{PALAVRAS-CHAVE}

Idosos. Evasão. Atividade física.

1 Pesquisa realizada com apoio financeiro da Coordenação de Aperfeiçoamento de Pessoal de Nível Superior (CAPES).

${ }^{2}$ Universidade Salgado de Oliveira. 


\section{ABSTRACT}

The study observed evasion of elderly in physical activity projects of the Department of Sport and Recreation, City of Rio das Ostras. Motivation is what leads the elderly to enroll in physical activity programs. After joining, the weight of motivation will cause it to remain or not in the activity. The avoidance of elderly in physical activity has been the subject of few studies, the focus of research is more directed to the motivation for membership. The research field was the 'Good Morning' and projects 'water aerobics'. Research indicated a quantitative and qualitative relationship between motivation and evasion, the main cause of evasion is the same as motivates the registration, health or lack of it.

\section{KEYWORDS:}

Elderly. Evasion. Physical activity. 


\section{INTRODUÇÃO}

As limitações funcionais decorrentes do avanço da idade do ser humano têm sido objeto de investigação recorrente. Desde o princípio da humanidade civilizada, indivíduos tentam fugir, ou ao menos amenizar, os efeitos do envelhecimento, fenômeno inevitável que avança em paralelo ao adiantamento etário. Fórmulas milagrosas indicadas como 'fontes da juventude' foram descobertas e negadas em seguida, ao passo que o envelhecimento saudável vem sendo o foco de muitos estudos, por despertar grande interesse de pesquisadores, graças ao evidente aumento da população idosa.

Segundo dados dos censos demográficos do Instituto Brasileiro de Geografia e Estatística (IBGE) em 1960, 3,3 milhões de brasileiros tinham 60 anos ou mais e representavam 4,7\% da população. Em 2010 a fatia etária idosa passou de 20,5 milhões de pessoas, $10,8 \%$ da população. $O$ envelhecimento no Brasil é tão evidente que os critérios de divisão dos dados dos censos mudaram. Até 1960 todas as pessoas com 70 anos ou mais eram colocadas na mesma categoria, hoje, a representatividade numérica desse grupo é tanta que as faixas etárias foram divididas a partir dos 70 anos em grupamentos de cinco em cinco anos até os 100 anos. Em decorrência do aumento demográfico significativo no Brasil, a população idosa se tornou foco de estudos, dada a preocupação em alinhar os modos e os hábitos desse nicho com um modo de vida mais saudável e digno, conforme preconizado pela Política Nacional da Saúde da Pessoa Idosa (BRASIL, 2006).

Justamente pelo processo irreversível descrito, o envelhecimento é um tema atual na sociedade, carecendo de constantes pesquisas para proporcionar aprofundamentos e inovações. Políticas públicas direcionadas aos idosos não são cumpridas, o que não deveria ocorrer no Brasil, que visivelmente, e estatisticamente, tem sua população mais envelhecida a cada ano. Apesar dos estudos e políticas voltadas ao bem estar dos idosos, ainda não vemos na prática esses indivíduos gozarem de qualidade de vida apropriada.

Nesse cenário então, a população idosa se destaca como um grupo em potencial, com necessidades específicas, exigindo qualificação especializada dos profissionais que atuam junto a esse público. Algumas iniciativas vêm ocorrendo há algumas décadas, principalmente no que se refere à atividade física direcionada a essa faixa etária, com intuito de retardar ou amenizar consequências indesejadas no organismo humano, ocasionadas pelo processo natural de envelhecimento.

Há uma posição consensual quanto aos benefícios trazidos pela prática regular de exercícios, como o controle do peso corporal, da pressão arterial e do nível insulínico, melhora na flexibilidade, na resistência física, na força muscular etc. Nas esferas psicológica e social os benefícios são também inquestionáveis: alívio do estresse, diminuição da depressão, o aumento da autoestima e redução do isolamento social. Esses são alguns dos principais efeitos benéficos da prática regular de atividades físicas que atingem qualquer indivíduo e, em especial o idoso. Estudos de Farinatti e Ferreira (2006), Lima-Costa e Veras (2003), Matsudo e Matsudo (1992) indicam detalhes desses benefícios e a divulgação dessas benesses é tão corriqueira nos meios de comunicação que parece ser já do senso comum.

Desde a criação da primeira 'universidade aberta à terceira idade', em 1973, em Toulouse, a importância das atividades físicas para pessoas aposentadas e idosas tem sido reconhecida. Desde então, as atividades físicas são consideradas parte destacada do currículo na maior parte dessas 'universidades', tanto em países do Primeiro quanto do Terceiro Mundo (FARIA JUNIOR, 2000 apud FARIA JUNIOR, 2002, p. 24).

Entretanto, segundo Faria Junior (2002), a atividade física como um campo de estudo e pesquisa veio se consolidar posteriormente. $O$ autor afirma que somente no início da década de 1990, constatou-se uma expansão mundial da pesquisa sobre a atividade física para idosos. Entre os acontecimentos que mais contribuíram para esta expansão destacam-se a criação do European Group for Research into Elderly and Physical Activity (Egrepa), em 1991, o aparecimento do Journal of Aging and Physical Activity (Japa), em 1993, e a realização da First International Conference of Egrepa, que teve lugar em Oeiras (Portugal), em 1993. 
Atualmente diversos autores se dedicam ao estudo do envelhecimento saudável e criaram definições que esclarecem os pormenores desse fenômeno. Segundo Spirduso, Francis e McRae (2005), o envelhecimento tem sido estudado como um processo inerente a todos os seres vivos e que se caracteriza pela perda da adaptação e pela contínua e progressiva deterioração dos tecidos e sistemas fisiológicos com consequências ao nível da sua funcionalidade e saúde.

Mazzeo e colaboradores (1998) entendem o envelhecimento como um processo complexo que envolve inúmeras variáveis como genética, estilo de vida e doenças crônicas, que interagem entre si e influenciam a forma como se envelhece. Embora seja visto como fenômeno irreversível que faz parte do amadurecimento humano, o envelhecimento tem causas ainda desconhecidas, e segundo Fonseca (2001 apud FARIA; MARINHO, 2004, p. 93) trata-se de "um fenômeno puramente fisiológico, que resulta o esgotamento da própria vitalidade celular, [...] aparecendo como consequência de várias manifestações patológicas (infecciosas, tóxicas e outras) que o cérebro vai suportando".

Neste sentido, o aumento da vulnerabilidade, as alterações fisiológicas e a diminuição da capacidade de resposta e de adaptação ao meio, característicos deste período de idade avançada, tornam esta fase alvo de importantes preocupações interdisciplinares, particularmente de interesse médico, psicológico e sociológico (FONSECA, 2001 apud FARIA; MARINHO 2004).

Baseado nas afirmações sobre o incontestável envelhecimento da população e nas pesquisas sobre os benefícios da atividade físicas, pesquisadores desenvolvem estudos sobre a relação entre atividade física, qualidade de vida e saúde no envelhecimento. Segundo Gonçalves, Duarte e Santos (2001) muitos são os autores que relacionam os benefícios sociais, psicológicos e físicos da atividade física para os indivíduos idosos, os benefícios conhecidos da atividade regular evidenciam que a magnitude da atividade física e do exercício tem sido retratada como um 'recurso de sobrevivência' para os adultos idosos, relacionado à autoeficiência.

Faria Junior (1999) cita vários estudos que mostram a relação positiva entre o envelhecimento e a atividade física. Destacam-se os estudos de revisão de Dishmam, que demonstram a redução do risco da depressão com o aumento do exercício.

Trabalhos de Guimarães e Caldas (2006), Lima-Costa e Veras (2003), Rocha e Freire (2007), Mazo, Mota e Gonçalvez (2005), Rigo e Teixeira (2005), Matsudo, Matsudo e Barros Neto (2001) vêm corroborar essa relação entre saúde do idoso e a prática da atividade física.

A dependência é o que menos esperamos desenvolver em idosos, não só nós da educação física, mas todos os profissionais que atuam frente a esse nicho. Todo tempo buscamos desenvolver atividades de possam estimular o idoso a executar suas tarefas diárias sem ajuda de familiar ou cuidador, assegurando a ele autonomia e independência. Tarefas simples como se calçar, se vestir e se alimentar por vezes são cerceadas em indivíduos de idade avançada que se entregam ao sedentarismo e à depressão.

Busca-se, além de promover a independência, conscientizar o idoso de que o envelhecimento é uma fase natural de sua evolução, que traz a reboque algumas alterações fisiológicas degenerativas, que os efeitos podem sim ser amenizados por meio de determinadas práticas, como por exemplo, a prática de atividade física. Assim o envelhecimento não deve ser encarado como uma fase decadente geradora de pessoas inválidas. Essa tomada de consciência permite que o idoso forme uma imagem melhorada de si mesmo e um aumento de sua autoestima, fato esse que lhe trará mais vontade de viver, e consequentemente, se exercitar, desencadeando um ciclo muito favorável a uma longa sobrevida (FARINATTI; FERREIRA, 2006).

É nesse viés que a atuação do professor de Educação Física se faz primordial, no esclarecimento dos efeitos naturais do envelhecimento, nas atitudes atenuantes e agravantes desse processo. Após a tomada de consciência, cabe a tarefa da administração das atividades adequadas ao público idoso, da criação de programas, promoção destes para buscar a adesão e perícia no dia a dia para evitar a evasão.

Para que se entenda o que motiva o idoso a praticar atividade física, assim como o que provoca sua evasão, é importante a apropriação de alguns conceitos sobre motivação humana. 
Quando discutimos motivação, relacionamos o homem e suas necessidades fisiológicas e psicológicas. Bara Filho e Miranda (1998) afirmam que a palavra chave para motivação é 'motivo', que significa o desejo de satisfazer alguma necessidade. A motivação é fundamental e essencial para que um indivíduo alcance seus objetivos. Sofre variações de indivíduo para indivíduo, pois há necessidades, objetivos e valores sociais distintos para cada um, variando ainda de acordo com o tempo histórico (MATSUDO; MATSUDO, 1992).

Pode-se aferir das teorias de motivação, que talvez a adesão em programas de atividade física gerada por uma motivação intrínseca possa ser mais duradoura que uma sugerida por terceiros, gerada por uma motivação extrínseca, pois um motivo interno ou pessoal tem grande chance de perdurar. Ao contrário, uma motivação externa, pode cessar, deixando o indivíduo propenso a interrupções ou desistências.

A motivação é o que mobiliza o idoso a praticar sua atividade, que deve ser capaz de satisfazer suas necessidades próprias ou a uma solicitação e/ou indicação de terceiros. Essa motivação, que pode ser de várias ordens, é o que leva o idoso à adesão em programas de atividades físicas, porém, após a adesão, o peso dessa motivação vai fazer com que ele permaneça ou não na atividade. A evasão de idosos em programas de atividades físicas já foi alvo de alguns estudos, mas o foco das pesquisas parece estar mais direcionado a motivação para a adesão, dado o número de estudos que encontramos sobre as duas temáticas.

A experiência do pesquisador deste estudo como professor atuante em projetos de atividade física voltada para idosos o fez perceber a grande incidência da evasão desses idosos nesses projetos, que despertou interesse e necessidade de buscar explicações para essas interrupções. Assim o problema do estudo centrou-se na evasão dos idosos nos programas públicos de atividades físicas de Rio das Ostras, Cidade que obteve grande crescimento populacional nos últimos anos, experimentando também aumento da parcela de idosos no total de habitantes.

\section{MÉTODO}

O objetivo do estudo foi analisar a motivação dos idosos, tendo como foco central os motivos para evasão dos mesmos em programas de atividades físicas.

O campo de pesquisa abrangeu os projetos de atividades físicas para idosos da Secretaria de Esportes e Lazer da Prefeitura de Rio das Ostras, especificamente o 'Bom Dia' e a Hidroginástica' devido a grande quantidade de idosos matriculados. Os pesquisados foram os idosos matriculados no ano de 2013 e que evadiram no último trimestre do mesmo ano.

Após feito um levantamento dos idosos participantes nos dois projetos, se deu continuidade ao trabalho de campo em visitas aos núcleos onde se desenvolvem os projetos, para comparação entre o cadastro de matriculados e as listas de presença dos professores de cada núcleo. Foi considerado evadido o participante que esteve ausente das aulas ao menos um mês a partir de outubro de 2013. O critério de inclusão para os sujeitos pesquisados foi o de indivíduos com 60 anos ou mais (classificação da Organização Mundial de Saúde para países em desenvolvimento).

Todos os pesquisados foram selecionados pelo critério de serem evadidos de projetos específicos. Para Costa Neto (1977) normalmente amostras não probabilísticas são utilizadas em pesquisa quando há uma restrição de cunho operacional ao uso da amostragem probabilística, como por exemplo o fato de não se ter acesso a todos os elementos da população.

Após detectar os alunos evadidos, o próximo passo da pesquisa foi buscar no cadastro de matrículas o contato telefônico dos alunos considerados evadidos, aos quais foi aplicado o instrumento de coleta elaborado para o estudo (apêndice A).

Optou-se por uma abordagem de caráter misto entre quantitativa e qualitativa. Segundo Goode e Hatt (1977 apud RICHARDSON, 1985) a pesquisa moderna deve rejeitar como uma falsa dicotomia a separação entre 'estudos qualitativos' e 'quantitativos', ou entre ponto de vista 'estatístico' e 'não estatístico'.

Foi utilizado como instrumento o questionário, que para May (2004) pode ser conduzido principal- 
mente através de três formas: a) por correspondência; b) por telefone; c) aplicação pessoal agendada. Dentre as propostas de May a escolhida para o questionário foi a telefônica pela economia, rapidez e viabilidade, já que estes pesquisados não frequentam mais os projetos, além da certeza do número maior de participantes, já que por correspondência poucos questionários são respondidos.

As ligações telefônicas foram gravadas por intermédio de aparelho marca Sony, modelo ICD-PX312 acoplado ao telefone em 'viva voz'. Estas gravações contêm a apresentação dos objetivos da pesquisa, um termo de consentimento livre e esclarecido (TCLE) adaptado a uma ligação telefônica que foi lida, como um 'aceite' em participar e colaborar como voluntário e a aplicação do questionário de coleta de dados (Apêndice A). A intenção da pesquisa, assim como a intenção do questionário foram explanadas com detalhes antes de se iniciar a aplicação em cada respondente, pois

[...] normalmente, o propósito do questionário deve ser explicado no início, de modo que as pessoas sintam-se envolvidas com o que você está fazendo. A pergunta de abertura também deve deixar as pessoas à vontade (MAY, 2004, p. 131).

Para elaboração do instrumento metodológico, foram observados estudos anteriores da mesma temática de vários pesquisadores da área, como o de Borges e Moreira (2009), Cerri e Simões (2007) e Cardoso e colaboradores (2008).

O modelo de instrumento baseado nos trabalhos citados, e depois de reestruturados pelo pesquisador, foram avaliados por três doutores experts na temática e sofreram adaptações em função das considerações tecidas por esses profissionais.

Para realização de todos os procedimentos que envolveram seres humanos no presente estudo, foram seguidas as normas da legislação específica. Incluem-se as gravações do contato telefônico que apresentam um termo de consentimento livre e esclarecido (TCLE) adaptado a uma ligação telefônica, ditado e acordado, onde o pesquisado afirma estar ciente da participação no estudo como voluntário e da garantida de sua proteção e privacidade.
A ética nessa pesquisa foi aprovada pelo Comitê de Ética da Universidade Salgado de Oliveira com número C.A.A.E. 26645914.3.0000.5289, parecer $n^{\circ}$ 689.285

\section{RESULTADOS E DISCUSSÃO}

Quanto ao questionamento referente à motivação inicial, embora esse estudo não esteja muito focado em resultados estatísticos, ele foi moldado sob uma perspectiva mista entre quantitativa e qualitativa, que nos permite afirmar que a maioria dos idosos pesquisados procurou a atividade física por vontade própria em busca de melhorias na saúde ou por indicação médica para amenizar problemas de saúde como bronquite asmática, diabetes, hipertensão, depressão, mialgias e problemas ósseos ou articulares, o que nos leva a concluir que a saúde é a principal motivação do idoso para a atividade física, como uma busca pessoal ou indicação médica. Então, a saúde é a principal motivação seja intrínseca ou extrínseca. Isso vai ao encontro do estudo de Cerri e Simões (2007) que afirma "(...) que a maioria (58\%) diz ter iniciado a hidroginástica seguindo ordens médicas" (p.87). Corrobora também com trabalho de Ribeiro e colaboradores (2012) que encontrou que a maioria dos idosos busca evitar ou prevenir problemas de saúde ou pratica atividade física porque o médico aconselhou. Encontraram também a saúde como principal motivação para a atividade física entre idosos os estudos de Freitas e colaboradores (2007), Gomes e Zazá (2009).

Em nosso estudo. além da saúde, apareceram em menor escala como motivação para a matrícula no 'Bom Dia' e Hidroginástica':

- a convivência em grupos e/ou colegas;

- praticar esportes;

- a alegria e saúde 'observados' nos participantes dos projetos;

- ideia de manter-se ativo;

- indicação de amigos praticantes dos projetos;

- busca de qualidade de vida e bem estar,;

- acreditar ser a prática de atividades físicas essencial para idosos;

- acompanhar a cônjuge na atividade; 
- busca de companhia (amigos);

- busca da vida saudável;

- distração.

Nas causas enumeradas acima, em quase todos os casos, essas apareceram relatadas pelos idosos associadas à saúde. Esse fato reafirma essa causa como a principal motivação para a matrícula nos projetos.

Não há muitos estudos já divulgados objetivando desvendar os motivos que fazem com que os idosos interrompam a prática de atividades físicas em projetos voltados a eles, mas podemos evidenciar alguns como a pesquisa de Cardoso e colaboradores (2008), que objetivou investigar os fatores que influenciam na adesão e desistência em um programa de exercício físico para idosos implantado nas Unidades Locais de Saúde (ULS) de Florianópolis, SC. Foram entrevistados 13 idosos, com idade média de 71,08 $(D P=5,75)$ anos, por meio de uma entrevista semiestruturada aplicada via telefone. Os motivos de desistência encontrados por Cardoso e colaboradores (2008) foram agrupados em três categorias. A categoria mais recorrente foi a das causas pessoais (problemas de saúde do cônjuge, morte do mesmo ou demais familiares). A segunda categoria relacionada pelos pesquisados foi saúde (cirurgias, tratamentos de doenças como labirintite e hérnia de estômago). E por último temos o próprio exercício físico, que geraram dores articulares sentidas após as aulas realizadas com exercícios realizados nas posições sentada e deitada.

Santariano, Haight, e Tager (2000 apud CARDOSO et al., 2008) se utilizaram de 2.046 pessoas acima de 55 anos (apesar da definição de 'idoso' da OMS ser de pessoa com 65 anos ou mais em países desenvolvidos e 60 anos ou mais em países em desenvolvimento e subdesenvolvidos) e verificou que as muIheres apontaram maior número de barreiras que os homens para realizar atividades físicas. Entre as barreiras de maior ocorrência estavam: a falta de companhia, a falta de interesse, a fadiga e os problemas de saúde. Nesse mesmo estudo ainda foi indicado que as razões ligadas à saúde aumentaram com a idade, e que nas pessoas mais idosas (>75 anos) os problemas de saúde e medo de quedas foram as barreiras mais citadas.
No estudo de Mazo, Cardoso e Aguiar (2006) aparecem os seguintes motivos: o estado de saúde; o medo de quedas e das suas consequências; a falta de motivação ou de força de vontade. No terceiro estudo, Dergance e colaboradores (2003 apud CARDOSO et al., 2008), avaliaram a percepção de idosos sedentários de diferentes etnias (mexicano-americanos e europeu-americanos), e observaram que a falta de interesse, falta de disciplina, de companhia, de conhecimento e não gostar de fazer atividades físicas foram as barreiras que diferiram entre as etnias.

Mazo, Mota e Gonçalvez (2005) colocam que barreiras associadas à atividade física são propícias às intervenções, uma vez que o reconhecimento destas pode guiar os programas e suas ações, de forma a definir metodologias e estratégias mais eficientes.

Em nosso estudo foram encontradas variadas causas que impedem os idosos de continuar nos projetos, mas a principal é a saúde, ou mais especificamente os problemas de saúde. Deixemos claro que não há intenções de medir nada estatisticamente, mas esse dado se faz importante e poderá dar esclarecimentos importantes quando relacionado a dados de motivação, 23 idosos (as) entre 47 pesquisados (as), indicaram problemas de saúde como causas da evasão dos projetos. Foram citados fraturas, cirurgias, torsões, artrose, reumatismos, esporão de calcâneo, hérnia de hiato, pneumonia, labirintite e tratamento de leucemia. Um agravante é que muitos desses idosos (as) se tratam em outras cidades por cobertura de plano de saúde, em muitos casos são inativos que após a aposentadoria se mudaram par Rio das Ostras, porém o plano de saúde cobre a sua cidade de origem. Além de doenças do próprio praticante ainda encontramos casos de doença e cirurgia do cônjuge ou de parente próximo.

Esses dados encontrados corroboram o estudo de Cardoso e outros (2008) que dividiu as causas de desistência em três categorias principais: 1) pessoais - problemas de saúde ou morte do cônjuge ou de outros familiares; 2) saúde - cirurgias, tratamentos de doenças como labirintite e hérnia de estômago; 3) exercício físico - dores articulares sentidas após as aulas realizadas com exercícios realizados nas posições sentada e deitada. Categorias que acabam por estarem relacionadas à saúde ou falta de condições 
saudáveis próprias ou de próximos que dependem de atenção do participante. Cardoso e colaboradores no mesmo estudo ainda apontam trabalhos de Santariano, Haight e Tager (2000 apud CARDOSO et al., 2008), Dergance e outros (2003 apud CARDOSO et al., 2008) e Mazo (2003 apud CARDOSO et al., 2008) que também apresentaram problemas de saúde como causas da evasão de idosos.

Surgiram em menor escala como motivos da evasão: cuidar de netos (as), piscina da hidroginástica profunda, horário da aula muito tardio, tomar conta de obras em casa, troca de projeto por conveniências diversas, viagem para visitar a família, acidente de carro, quantidade excessiva de alunos nas aulas, praia cheia no horário da atividade (Bom Dia), plantão do trabalho, horário da aula coincide com o da faculdade que cursa. Um idoso informou desconforto gástrico e enjoo nos exercícios em decúbitos, posição que representa metade da aula do 'Bom Dia'.

\section{CONSIDERAÇÕES FINAIS}

Se estabelece assim alguma relação entre a motivação para a matrícula e as causas da evasão dos idosos em sua atividade física, segundo vários estudos sobre a adesão na atividade física é a saúde ou a falta dela que os motiva a iniciar as atividades, é o que tira os idosos de suas casas em busca de se exercitar. Porém, percebemos também, que a motivação do idoso para se inserir em programas de atividade física, a saúde de uma forma geral, acaba por ser a principal e mais reincidente barreira para sua continuidade, ao menos em Rio das Ostras e nos locais onde os estudos anteriores aqui relacionados foram realizados.

A mídia noticia a todo tempo estudos sobre a necessidade da prática de exercícios para um enveIhecimento saudável, e isso se tornou do conhecimento de todos, tanto que uma geração que não foi habituada quando jovem a essa prática, hoje admite necessitar de atividade física nas suas diversas formas. Porém os idosos uma vez convencidos que necessitam de se exercitar, se inserem na prática, e problemas de saúde acabam por impedi-los, seja momentaneamente ou definitivamente.

Nós, professores que atuamos junto a esse público, já percebemos há muito tempo os alunos que se ausentam e após curto ou longo período retornam, mas nem sempre temos condições de consultar as causas da ausência, por quantidade de alunos e os vários locais onde atuamos. Esse estudo nos auxilia entendemos um pouco mais as indas e vindas dos idosos na prática de atividade física.

Talvez uma estratégia de acompanhamento dos resultados alcançados pudesse auxiliar na retenção. Uma forma de verificar se o que buscou-se em termo de melhora na saúde está sendo alcançado, saber se a atividade escolhida é capaz de proporcionar o esperado pela indicação do médico, ou superar a expectativa de bem estar do próprio participante.

\section{REFERÊNCIAS}

BARA FILHO, Maurício G.; MIRANDA, Renato. Aspectos psicológicos do esporte competitivo. Revista Treinamento Desportivo, Curitiba, v. 3, n. 3, p. 62-72, 1998.

BORGES, Milene R. D.; MOREIRA, Ângela K. Influências da prática de atividades físicas na terceira idade: estudo comparativo dos níveis de autonomia para o desempenho nas AVDs e AIVDs entre idosos ativos fisicamente e idosos sedentários. Revista Motriz, Rio Claro, v. 15 n. 3, p. 562-573, jul./set. 2009.

BRASIL. Ministério da Saúde. Portaria GM n².528, de 19 de outubro de 2006. Aprova a Política Nacional de Saúde da Pessoa Idosa - PNSI. Diário Oficial da União, Poder Executivo, Brasília, DF, 20 out. 2006. Disponível em: <http://bvsms.saude.gov.br/bvs/publicacoes/atencao_saude pessoa_idosa_envelhecimento_v12.pdf $>$ Acesso em $2 \overline{2}$ abr. 2014.

CARDOSO, Adílson S. et al. Fatores influentes na desistência de idosos em um programa de exercício físico. Revista Movimento, Porto Alegre, v. 14, n. 1, p. 225-239, jan./abr. 2008.

CERRI, Alessandra de S.; SIMÕES, Regina. Hidroginástica e idosos: por que eles praticam?. Revista Movimento, Porto Alegre, v. 13, n. 1, p. 81-92, jan./abr. 2007.

COSTA NETO, Pedro Luíz de O. Estatística. São Paulo: Edgard Blücher, 1977.

DERGANCE, Jeannae M. et al. Barries to and benefits of leisure time physical activity in the elderly: differences across cultures. In: CARDOSO, A. S. et al. Fatores influentes na desistência de idosos em um programa de exercício físico. Revista Movimento, Porto Alegre, v.14, n.1, p. 225239, jan./abr. 2008. 
FARIA JUNIOR, Alfredo G. de. Atividade física para idosos portadores e não portadores de deficiência visual: um estudo comparativo. Revista Brasileira de Ciências do Esporte, Campinas, v. 23, n.3, p. 23-34, jun. 2002. Disponível em: < http://rbceonline.org.br/revista/index.php/RBCE/article/view/282/265>. Acesso em: 22 abr. 2014.

Atividades físicas para idosos: um desafio para a educação gerontológica. In: Congresso de Educação Física e Ciências do Esporte dos Países de Língua Portuguesa, 7., Florianópolis, 1999. Anais... Florianópolis: [s.n.], 1999. p. $119-128,1999$.

FARINATTI, Paulo de Tarso V.; FERREIRA, Marcos S. Saúde, promoção da saúde e educação física. Rio de Janeiro: UERJ, 2006.

FONSECA, Fernandes A. Saúde mental e terceira idade. In: FARIA, Luísa; MARINHO, C. Actividade Física, saúde e qualidade de vida na terceira idade. Revista Portuguesa de Psicossomática, Lisboa, v. 6, n. 1, jan./jun. p. 93-104, 2004. Disponível em: $<$ http://redalyc.org/articulo. oa?id=28760113 > ISSN 0874-4696. Acesso em: 22 Abr. 2014.

FREITAS, Clara Maria S. M. et al. Aspectos motivacionais que influenciam a adesão e manutenção de idosos a programas de exercícios físicos. Revista Brasileira de Cineantropometria e Desempenho Humano, Florianópolis, v. 9, n. 1, p. 92-100, 2007.

GOOD, Willian J.; HATT, Paul K. Métodos em Pesquisa social. In: RICHARDSON, Roberto J. Pesquisa social: métodos e técnicas. São Paulo: Atlas, 1985.

GONÇALVES, Andréa. K.; DUARTE, Cátia. P.; SANTOS, Cristiane. L. dos. Atividade física na fase da meia-idade: motivos de adesão e de continuidade. Revista Movimento, Porto Alegre, v. 7, n. 15, p. 75-88, jul./dez. 2001. Disponível em: <http://www.redalyc.org/articulo.

oa?id=115318170007> . Acesso em: 22 abr. 2014.

GOMES, Kátia Virgínia.; ZAZÁ, Daniela Coelho. Motivos de adesão a prática de atividade física em idosas. Revista Brasileira de Atividade Física \& Saúde, Londrina, v. 14, n. 2, p. 132-138, mai./ago. 2009.

GUIMARÃES, Joanna M. N.; CALDAS, Célia P. A influência da atividade física nos quadros depressivos de pessoas idosas: uma revisão sistemática. Revista Brasileira de Epidemiologia, São Paulo, v. 9, n. 4, p. 481-492, dez. 2006.

INSTITUTO BRASILEIRO DE GEOGRAFIA E ESTATÍSTICA. Censo Demográfico 2010. Rio de Janeiro, 2011.

LIMA-COSTA, Maria Fernanda; VERAS, Renato P. Saúde pública e envelhecimento. Caderno de Saúde Pública, Rio de Janeiro, v. 19, n. 3, p. 700-701, mai./jun. 2003.
MATSUDO, S. M.; MATSUDO, V. K. R. Prescrição e benefícios da atividade física na terceira idade. Revista Brasileira de Ciência e Movimento, São Caetano do Sul, v. 6, n. 4, p. 19-30, out./dez. 1992.

MAZO, G. Z.; MOTA, J. A. P. S.; GONÇALVEZ, L. H. T. Atividade física e qualidade de vida de mulheres idosas. Revista Brasileira Ciências do Envelhecimento Humano, Passo Fundo, v. 2, n. 1, p. 115-118, jan./jun. 2005.

MATSUDO, Sandra M.; MATSUDO, Vítor K. R.; BARROS NETO, T. L. Atividade física e envelhecimento: aspectos epidemiológicos. Revista Brasileira de Medicina do Esporte, São Paulo, v. 7, n. 1, p. 2-13, jan./fev. 2001.

MAY, Tim. Pesquisa social: questões, métodos e processos. 3. ed. Porto Alegre: Artmed, 2004.

MAZO, Giovana Z. Atividade Física e Qualidade de Vida de Mulheres Idosas. 218 p. Tese (Doutorado em Educação Física). Faculdade de Ciências do Desporto e de Educação Física - Universidade do Porto, Porto, 2003 In: CARDOSO, Adílson S. et al. Fatores influentes na desistência de idosos em um programa de exercício físico. Revista Movimento, v.14, n.1, p. 225-239, jan./abri. 2008.

MAZO, Giovana Z.; CARDOSO, Fernando L.; AGUIAR, Daniela L. D. Programa de hidroginástica para idosos: motivação, auto-estima e auto-imagem. Revista Brasileira de Cineantropometria e Desempenho Humano, Florianópolis, v. 8 , n.2, p. 67-72, abr./jun. 2006.

MAZO, Giovana Z.; MOTA, Jorge A. P. S.; GONÇALVEZ, Lúcia H. T. Atividade física e qualidade de vida de mulheres idosas. Revista Brasileira Ciências do Envelhecimento Humano, Passo Fundo, v. 2, n. 1, p. 115-118, jan./jun. 2005.

MAZZEO, Robert. et al. Exercise and physical activity for older adults. Medicine \& Science in Sports \& Exercise, Auburn, v. 30, n. 6, p. 1510-1530, jun. 1998.

RIBEIRO, José Antônio B. et al. Adesão de idosos a programas de atividade física: motivação e significância. Revista Brasileira de Ciências do Esporte, Florianópolis, v. 34, n. 4, p. 969-984, out./dez. 2012.

RIGO, Maria de L. N. R.; TEIXEIRA, Denílson C. Efeitos da atividade física na percepção de bem-estar de idosas que residem sozinhas e acompanhadas. Revista Unopar Científica Ciências Biológicas e da Saúde, Londrina, v. 7, n. 1, p. 13-20, out. 2005.

ROCHA, Saulo V.; FREIRE, Malú O. Nível de atividade física habitual e autopercepção do estado de saúde em idosas no município de Jequié, Bahia. Revista Brasileira Promoção de Saúde, Fortaleza, v. 20, n. 3, p. 161-167, jul./set. 2007. Disponível em: <http://ojs.unifor.br/index.php/RBPS/article/ view/1020>. Acesso em: 22 abr. 2014. 
SANTARIANO, Willian A.; HAIGHT, Thaddeus J.; TAGER, Ira $B$. Reasons given by older people for limitation or avoidence of leisure time physical activity. In: CARDOSO, Adílson S. et al. Fatores influentes na desistência de idosos em um programa de exercício físico. Revista Movimento. Porto Alegre, v.14, n.1, p. 225-239, jan./abr. 2008.
SPIRDUSO, Wannen W.; FRANCIS, Karen; MCRAE, Priscilla G. Physical dimensions of aging. 2. ed. Champaign: Human Kinetics, 2005.

WORLD HEALTH ORGANIZATION. Active ageing: a police framework. Madrid, 2002. Disponivel em: <www.who. org >. Acesso em: 18 maio 2013. 